\title{
Amniocentesis precoz: estudio citogenético en 505 embarazadas
}

\author{
Luz Mery Bernal Parra', Thomaz Rafael Gollop², Nadyr Naccache².
}

\begin{abstract}
1. Bióloga, Pontificia Universidad Católica Do Paraná. Magister en Biología celular y Genética, Pontificia Universidad Javeriana.Doctorado en Biología Genética, Universidad De Sao Paulo. Docente Asistente, UNAD.

2. Serviço de Ginecologia e Genética de São Paulo, Brasil

Correspondencia: luz.bernal@unad.edu.co
\end{abstract}

Recibido: 11/11/2011 - Aceptado: 20/12/2011

\begin{abstract}
Resumen
El diagnóstico citogenético en líquido amniótico obtenido por amniocentesis precoz (AP) es una alternativa a la embarazada de primer trimestre. El objetivo de este trabajo fue investigar el tipo y la frecuencia de anormalidades cromosómicas encontradas en muestras de líquido amniótico obtenido por medio de esa técnica de colecta. En un periodo de 5 años, 53। AP fueron realizadas en 505 pacientes (479 gestaciones únicas y 26 gemelares), con edad gestacional variando de 12 0/7 a 14 6/7 semanas. La principal indicación para la realización del procedimiento fue edad materna avanzada (66,3\% de los casos). Los cultivos fueron establecidos en medio Chang y Amniomax (cultivo en frasco). El tiempo de colecta para la obtención de las preparaciones citogenéticas varió de 7 a 22 días ( 1 5,5 \pm 2,8). Por lo menos 25 células fueron analizadas por paciente. El tiempo de entrega de resultados varió de 12 a 25 días ( $18,7 \pm 2,8$ días). La frecuencia de éxito de cultivo fue del 98,7\%. Cariotipos anormales fueron identificados en 22 casos (4,2\%), siendo tres $(0,6 \%)$ rearreglos cromosómicos equilibrados y 19 (3,6\%) anomalías cromosómicas no equilibradas. De estas, la anormalidad cromosómica más frecuente fue la trisomía del cromosoma 21 (27,3\%). Fueron identificados cinco casos de cromosomas marcadores, tres en forma de mosaico. Fueron confirmados seis (18,2\%) resultados anormales detectados en Vellosidad Corial (VC). La frecuencia de pseudomosaicismo encontrada fue del 2,3\%. Nuestros datos confirman la precisión del estudio citogenético en líquido amniótico obtenido por AP. Con procedimientos de rutina bien establecidos y protocolos adecuadamente estandarizados en el laboratorio de diagnóstico prenatal, se aumenta el éxito de los cultivos de células de líquido amniótico obtenido por AP y se reduce el tiempo medio de entrega de resultados.
\end{abstract}

Palabras clave: diagnóstico prenatal, amniocentesis precoz, anomalías cromosómicas.

\section{Abstract \\ The early amniocentesis: cytogenetic study in 505 pregnant}

Cytogenetic diagnosis in amniotic fluid after early amniocentesis is an alternative to woman in the first trimester of pregnancy. The aim of this study was to ascertain the type and frequency of chromosomal aberrations in amniotic fluid samples collected after early amniocentesis (EA). In the period of five years, 53। EA were carried out in 505 patients (479 single and 26 multiple gestations) the gestational age of whom varied between 12 $+0 / 7-14+6 / 7$ weeks + days. The main indication for offering the procedure was advanced maternal age $(66.3 \%$ cases). Cultures were established in Chang or Amniomax media, using flask incubation. The time to obtain the cytogenetic preparations ranged from 7 to 22 days (I 5,5 $\pm 2.8 \mathrm{~m} \pm$ s.d.). At least 25 cells 
were analyzed per patient. The time to provide the cytogenetic results ranged from 12 to 25 days ( $18.7 \pm$ 2.8 days, $m \pm$ s.d). The culture success rate was 98.7\%. Abnormal karyotypes were identified in 22 cases (4.2\%); three (0.6\%) of these were balanced and 19 (3.6\%) were unbalanced chromosomal rearrangements. The most frequent unbalanced chromosomal aberration was trisomy 21 (27.3\%). Five cases of marker chromosomes were identified, three of which were mosaic. Six abnormal results found in chorionic villus sampling were confirmed. The frequency of pseudomosaicism was $2.3 \%$. Our data confirm the accuracy of cytogenetic studies on amniotic fluid collected through EA. When established routine procedures and standardized protocols are used, the incidence of culture success increases significantly, and the mean time to deliver the results decreases.

Keywords: prenatal diagnosis, early amniocentesis, chromosomal abnormalities.

\section{Resumo \\ Amniocentese precoce: estudo citogenético em 505 gestantes}

O diagnóstico citogenético em líquido amniótico obtido por amniocentese precoce (AP) é uma alternativa à gestante de primeiro trimestre. $\bigcirc$ objetivo deste trabalho foiaveriguar o tipo e a frequência de anormalidades cromossômicas encontradas em amostras de líquido amniótico obtido por meio dessa técnica de coleta. Num período de 5 años, 53 I AP foram efetuadas em 505 pacientes (479 gestações únicas e 26 gemelares), com idade gestacional variando de 12 0/7 a 14 6/7 semanas. A principalindicação para a realização do procedimento foi idade materna avançada (66,3\% doscasos). As culturas foram estabelecidas em meio Chang e Amniomax (cultura em frasco). $\bigcirc$ tempo de coleta para a obtenção das preparações citogenéticas variou de 7 a 22 dias ( 15,5 $\pm 2,8)$. Pelo menos 25 células foram analisadas por paciente. $\bigcirc$ tempo de entrega deresultados variou de 12 a 25 dias (18,7 $\pm 2,8$ dias). A frequência de sucesso de cultura foi de 98,7\%. Cariótipos anormais foram identificados em 22 casos (4,2\%), sendo três (0,6\%)rearranjos cromossômicos equilibrados e 19 (3,6\%) anomalias cromossômicas nãoequilibradas. Destas, a anormalidade cromossômica mais freqüente foi atrissomia docromossomo 2 I (27,3\%). Foram identificados cinco casos de cromossomos marcadores, três em forma de mosaico. Foram confirmados seis ( $18,2 \%)$ resultados anormais detectados em AVC. A freqüência de pseudomosaicismo encontrada foi de 2,3\%. Nossos dados confirmam a precisão do estudocitogenético em líquido amniótico obtido por AP. Com procedimentos de rotina bem estabelecidos e protocolos adequadamentepadronizados no laboratório de diagnóstico pré-natal, aumenta-se o sucesso das culturas de células de liquido amniótico obtido por AP e reduz-se o tempo médio de entrega de resultados.

Palavras-chave: diagnóstico pré-natal, amniocentese precoce, anomalias cromossômicas.

\section{Introducción}

Las anomalías cromosómicas son causas importantes de mortalidad perinatal y morbidez infantil. El riesgo de aberraciones cromosómicas constituye la más frecuente indicación para realizar un procedimiento prenatal invasivo. El objetivo de las técnicas de diagnóstico citogenético prenatal es proporcionar a la pareja y a su médico, información del embarazo para que decidan cómo será la asistencia durante el transcurso del mismo.

Un diagnóstico precoz se constituye en una ventaja para el rastreo de anomalías cromosómicas en el primer trimestre de la gestación. Así, se vuelve menos traumática una eventual interrupción por la anomalía diagnosticada. La amniocentesis precoz (AP) representa una técnica alternativa, capaz de 
proporcionar resultados comparables a los de la amniocentesis tradicional (AT) en términos de seguridad y calidad de las preparaciones citogenéticas.

El éxito del cultivo en AP depende de la edad gestacional y se torna satisfactorio a partir de la 11a semana (1). Después de ese periodo, el material recolectado permite diagnosticar con la rapidez y precisión que exige el diagnóstico citogenético (2, $3,4)$.

En Brasil, la AP fue introducida en 1992 y, desde entonces, se ha reportado una frecuencia de alrededor del 99\% en el éxito del cultivo $(5,6)$.

Este trabajo analiza el tipo y la frecuencia de anormalidades cromosómicas encontradas en muestras de líquido amniótico obtenido por medio de AP en un grupo de embarazadas atendido en una institución brasilera de diagnóstico prenatal.

\section{Materiales y métodos}

La población estudio fue constituida por embarazadas sometidas a AP para la realización del diagnóstico citogenético prenatal. En una consulta de consejería genética antes del procedimiento, se informó a la paciente acerca de las limitaciones, riesgos, beneficios y tiempo medio de obtención de los resultados. El día del examen, antes de la punción verificó por ultrasonografía la viabilidad fetal, la edad gestacional, la inserción de la placenta, el número de fetos y se evaluó el volumen del líquido amniótico (antes de la punción). Se recolectó aproximadamente $1 \mathrm{ml}$ de LA por semana gestacional. Excepto en los primeros 19 procedimientos, realizados con aguja 20 gauge, la punción fue realizada con aguja 22 gauge bajo visualización ultrasonográfica directa. La punción con aguja 20 gauge fue abandonada después de la recomendación de Henry y Miller (7).

Los amniocitos fueron cultivados por la técnica de cultivo en frasco, en presencia de CO2 (5\%) a $37^{\circ} \mathrm{C}$. Se utilizó medio Chang D (Irvine Sdentific) o Amniomax (Life Technologies). En media, la cosecha se produjo después de 15 días de cultivo. Se añadió colchicina(Sigma) al cultivo 50 minutos antes de la cosecha en una concentración de 4x10$5 \mathrm{M}$. Las células fueron hipotonizadas con citrato de sodio $(0,8 \%)$ por $25-30$ minutos a $37^{\circ} \mathrm{C}$ y fijadas con solución 3:1 de metanol-ácido acético (Merck). $\mathrm{El}$ análisis cariotípico fue realizado en preparaciones procedentes de dos o más frascos de cultivo. Fueron analizadas 25 metafases por paciente: diez con coloración Giemsa de rutina y 15 con bandas G.

Cuando en uno de los frascos de cultivo se encontró una o más células anormales, la diferenciación entre el mosaicismo verdadero y el pseudomosaicismo siguió el protocolo de Hus $(8,9)$. El mosaicismo verdadero fue definido como la presencia de diferentes complementos cromosómicos en dos o más linajes celulares provenientes de dos o más frascos de cultivo. El pseudomosaicismo fue definido como la presencia de una o varias células en un único frasco de cultivo, con un complemento cromosómico diferente de aquel encontrado en la mayoría de las células analizadas. La contaminación por células maternas (CCM) fue definida como la ocurrencia de por lo menos una célula XX en medio de linajes mayoritariamente XY.

En los casos de resultados que incluían rearreglos cromosómicos y cromosomas marcadores, fue realizado el estudio citogenético de los padres. Los cromosomas marcadores fueron estudiados a través de diferentes métodos de bandas cromosómicas ( $G$, C, NOR) y, en un caso, a través de hibridación in situ por fluorescencia (FISH). En los casos de resultados que incluían mosaicismo verdadero y o/cromosomas marcadores, se aconsejó a la paciente la confirmación del diagnóstico citogenético a través del análisis de sangre fetal (cordocentesis).

En AP realizadas para la confirmación de mosaicismo encontrado en VC, se utilizaron muestras de los tres frascos de cultivo y se analizaron entre 50 y 100 células.

El fracaso en los cultivos fue definido como la ausencia de crecimiento celular o contaminación. En esos casos, fue necesario repetir la amniocentesis. La frecuencia de éxito delcultivo fue definida como el número de casos en que el resultado citogenético fue obtenido por el número total de casos en que se inició un cultivo.

A partir del establecimiento del cultivo, el tiempo de colecta fue representado por el número de días necesarios para que la proliferación celular fuera suficiente para la obtención de un número razonable de metafases para análisis. Para verificar la existencia 
Tabla 1. Indicaciones para la realización de la amiocentesis precoz.

\begin{tabular}{|c|c|c|}
\hline INDICACIONES & TOTAL & $(\%)$ \\
\hline Edad materna avanzada $\geq 35$ ańos & 335 & 66.3 \\
\hline - $\quad$ Sin otras indicaciones & 286 & 56.6 \\
\hline - Con otras indicaciones & 49 & 9.7 \\
\hline Anomalía cromosómica previa & 6 & 1.2 \\
\hline Hijo anterior con malformación congénita & 4 & 0.8 \\
\hline Anomalía en ultrasonido & 6 & 1.2 \\
\hline Rearreglos cromosómicos en los padres & 2 & 0.4 \\
\hline Anomalía cromosómica en VC en mosaico & 17 & 3.3 \\
\hline Anomalía cromosómica en VC sin mosaico & 6 & 1.2 \\
\hline Abortos a repetición & 8 & 1.6 \\
\hline \multicolumn{3}{|l|}{ Edad materna $<35$ años } \\
\hline Ansiedad & 110 & 21.8 \\
\hline Anomalía cromosómica previa & 18 & 3.5 \\
\hline Hijo anterior con malformación congénita & 9 & 1.8 \\
\hline Anomalía en ultrasonido & 8 & 1.6 \\
\hline Anomalía cromosómica en VC en mosaico & 8 & 1.6 \\
\hline Anomalía cromosómica en VC sin mosaico & 2 & 0.4 \\
\hline Abortos a repetición & 9 & 1.8 \\
\hline Enfermedades congénitas & 6 & 1.2 \\
\hline TOTAL & $505^{*}$ & 100 \\
\hline
\end{tabular}

${ }^{*}$ con base en 505 pacientes

de correlación entre el volumen de líquido amniótico aspirado y el tiempo de colecta, fue realizado un análisis de regresión lineal.

El tiempo de entrega de los resultados fue el periodo transcurrido entre la realización de la amniocentesis y la entrega de los resultados citogenéticos.

Posteriormente, fueron enviados cuestionarios a las pacientes con la finalidad de obtener informaciones acerca de los datos obstétricos del parto y clínicos del recién nacido. El seguimiento de los casos fue realizado por teléfono (con la paciente o el obstetra) cuando esos datos no fueron remitidos a la institución donde este estudio fue conducido.

\section{Resultados}

En un período de cinco años, 531 AP fueron realizadas en 505 pacientes ( 479 gestaciones únicas y 26 gemelares). La edad gestacional en el momento de la colecta varió de 12 0/7 a 146/7 semanas (media \pm 1 d.p. $=13,7 \pm 0,67)$. La edad materna varió de $18 \mathrm{a}$ 46 años (media \pm 1 d.p. $=35,7 \pm 4,47$ ). En el grupo por encima de 35 ańos, la edad media fue de $38,3 \pm$ 2,41 años.

\section{Indicaciones}

La principal indicación para la realización del procedimiento fue la edad materna avanzada, representando un 66,3\% de los casos (Tabla 1). En los cariotipos anormales, diez (45,5\%) tuvieron cómo única indicación edad materna avanzada y diez $(45,5 \%)$ presentaron, además de la edad materna, otras indicaciones: translucencianucal aumentada $(n=2)$, hijo anterior malformado $(\mathrm{n}=1)$, rearreglo cromosómico en la madre $(n=1)$ y anomalía cromosómica en VC $(n=6)$. En un caso $(4,5 \%)$ la indicación para la realización del examen fue la presencia de un hijo anterior con síndrome de Down y, en otro, la confirmación de los resultados (mosaico) obtenidos en la VC.

Otras razones para la realización del examen están relacionadas en la Tabla 1 . La distribución de la edad gestacional en que los procedimientos fueron realizados, para cada categoría de edad materna, se encuentra en la Tabla 2. 
Tabla 2. Número de procedimientos realizados por edad gestacional, edad materna y algunas características de laboratorio.

\begin{tabular}{|c|c|c|c|c|}
\hline \multicolumn{5}{|c|}{ SEMANAS DE GESTACIÓN } \\
\hline Edad materna (años) & $12-12^{6 / 7}$ & $13-13^{6 / 7}$ & $14-14^{6 / 7}$ & TOTAL* \\
\hline$<30$ & 7 & 28 & 24 & $59(11.1 \%)$ \\
\hline $30-34$ & 15 & 66 & 34 & $115(21.7 \%)$ \\
\hline $35-39$ & 21 & 135 & 90 & $246(46.3 \%)$ \\
\hline$>40$ & 12 & 58 & 41 & $111(20.9 \%)$ \\
\hline TOTAL & $55(10.4 \%)$ & $287(54.0 \%)$ & $189(35.6 \%)$ & $531(100.0 \%)$ \\
\hline Medida vol. LA (mะ 1 d.p) & $13.7 \pm 2.2$ & $14.9 \pm 1.3$ & $145.0 \pm 1.5$ & $14.3 \pm 1.5$ \\
\hline Falla en el cultivo & $2(0.4 \%)$ & $3(0.6 \%)$ & $2(0.4 \%)$ & $1.3 \%$ \\
\hline Tiempo de colecta $(\mathrm{m} \pm 1$ d.p) & $15.7 \pm 3.4$ & $14.5 \pm 2.5$ & $14.2 \pm 2.8$ & $15.5 \pm 2.8$ \\
\hline \multicolumn{5}{|l|}{ Tiempo de entrega de } \\
\hline Resultados (mะ 1 d.p) & $19.5 \pm 2.6$ & $18.8 \pm 2.7$ & $18.2 \pm 2.9$ & $18.7 \pm 2.8$ \\
\hline
\end{tabular}

${ }^{*}$ con base en 531 procedimientos

\section{Procedimientos de laboratorio}

El tiempo de colecta para la obtención de las preparaciones citogenéticas varió de 7 a 22 días (m \pm 1 d.p. $=15,5 \pm 2,8$ ). Existe una correlación negativa de pequeña intensidad, sin embargo estadísticamente significativa, entre el volumen de líquido amniótico utilizado en el cultivo y el número de días necesarios para la obtención de las preparaciones: $\mathrm{y}=15,5-0,08$. $\mathrm{x}(\mathrm{F}=10,46 ; \mathrm{gl} 1=1 ; \mathrm{gl} 2=524 ; \mathrm{P}=0,001)$, coeficiente de correlación $\mathrm{r}=-0,14$ significativamente diferente de cero. El tiempo de entrega de resultados varió de 12 a 25 días ( $m \pm 1$ d.p. $18,7 \pm 2,8$ días).

La frecuencia de éxito en los cultivos fue del 98,7\%. Fueron registrados siete casos (1,3\%) de falla: cinco por falta de crecimiento celular y dos por contaminación. De los cinco casos de ausencia de crecimiento celular, tres ocurrieron dentro de los primeros diez procedimientos realizados, es decir, cuando la técnica estaba siendo implementada. Del total de siete casos, tres repitieron AP y en ellos se obtuvieron resultados citogenéticos en la segunda tentativa. Dos pacientes de las siete, repitieron el examen, pero ya se encontraban en la edad gestacional de la amniocentesis tradicional. En dos casos, no fue posible repetir el procedimiento; una de las pacientes volvió a su país de origen y otra optó por un aborto social.

\section{Análisis citogenético}

De los 531 procedimientos realizados, fueron obtenidos $527(99,3 \%)$ resultados citogenéticos.
Cariotipos anormales fueron identificados en 22 casos $(4,2 \%)$, siendo tres $(0,6 \%)$ rearreglos cromosómicos equilibrados y $19(3,6 \%)$ anomalías cromosómicas no equilibradas (Tablas 3, 4 y 5). De estas, la anomalía cromosómica más frecuente fue la trisomía del cromosoma $21(27,3 \%)$, seguida por la trisomía del cromosoma $18(18,2 \%)$ y por el cariótipo 47, XXY (13,6\%). Fueron identificados cinco casos de cromosomas marcadores, tres en forma de mosaico, Tabla 3.

\section{Cromosomas marcadores}

En el mosaicismo 46,XX/47,XX,+mar (paciente 11, Tabla 4), fue encontrado un marcador no satelitado, presente en $55 \%$ de las metafases, de tamaño menor al de los cromosomas del grupo G. El patrón de bandas $\mathrm{G}$ no permitió la identificación de este marcador. En la identificación por FISH fueron utilizadas sondas centromérícas (AneuScreen-Vysis) para los cromosomas X, Y, 18 (Figura 1). Se utilizaron también sondas Coatsome (Oncor) para los cromosomas 15, 22 y 9. Este análisis fue realizado en el Servicio de Consejería Genética de Botucatu IBB/UNESP (Figura 2 y 3 ). No fue posible demostrar el origen del marcador con las sondas utilizadas. La cordocentesis realizada en la 20a semana confirmó el mosaicismo. Los padres, cuyo cariotipo era normal, optaron por la interrupción del embarazo.

Un segundo feto presentó cariotipo en mosaico 46,XY/47,XY,+mar (paciente 12). El cromosoma 
Tabla 3. Resultados citogenéticos de 531 muestras recolectadas por amiosentesis precoz.

\begin{tabular}{lcc}
\hline RESULTADOS CITOGENÉTICOS & n & (\%) \\
\hline $46, X X$ & 269 & 50.6 \\
$46, X Y$ & 236 & 44.4 \\
Rearreglos equilibrados & 3 & 0.6 \\
Anomalías sin mosaico & 15 & 2.8 \\
Anomalías en mosaico & 4 & 0.8 \\
Falta de crecimiento / contaminación & 4 & 0.8 \\
Total & 531 & 100 \\
\hline
\end{tabular}

Tabla 4. Anomalías cromosómicas no equilibradas en amiosentesis precoz.

\begin{tabular}{|c|c|c|c|c|}
\hline Paciente & $\begin{array}{l}\text { Edad materna y } \\
\text { gestacional }\end{array}$ & INDICACION & $\begin{array}{c}\text { ANOMALIAS } \\
\text { CROMOSOMICAS NO } \\
\text { EQUILIBRADS (AP) }\end{array}$ & SEGUIMIENTO \\
\hline \multirow[t]{2}{*}{1} & $4314^{5 / 7}$ & Edad materna +AVC & $47, \mathrm{XX},+21$ & IG \\
\hline & & $(47, X X,+21)$ & & \\
\hline 2 & $3613^{1 / 7}$ & Edad materna & $47, X X,+21$ & IG \\
\hline 3 & $3613^{2 / 7}$ & Edad materna + TNA: $5 \mathrm{~mm}$ & $47, X X,+21$ & IG \\
\hline 4 & $3614^{1 / 7}$ & Edad materna & $47, X X,+21$ & $\mathrm{RN}$ afectado \\
\hline 5 & $4113^{2 / 7}$ & Edad materna +TNA: $5.7 \mathrm{~mm}$ & $47, X X,+21$ & IG \\
\hline \multirow[t]{2}{*}{6} & $4014^{6 / 7}$ & Edad materna +AVC & $47, X X,+21$ & IG \\
\hline & & $\{46, X Y 747, X Y,+21\}(11 \%: 89 \%)$ & & \\
\hline 7 & $4513^{0 / 7}$ & Edad materna + HAM & $47, X X,+18$ & Muerte neonatal \\
\hline \multirow[t]{2}{*}{8} & $3912^{5 / 7}$ & Edad materna +VC & $47, \mathrm{XY},+18$ & IG \\
\hline & & $\{46, \mathrm{XY} / 47, \mathrm{XY}+18\}(10 \%: 90 \%)$ & & \\
\hline 9 & $4112^{0 / 7}$ & Edad materna & $47, X Y,+18$ & IG \\
\hline \multirow[t]{3}{*}{10} & $4013^{2 / 7}$ & Edad materna $+\mathrm{VC}$ & $46, \mathrm{XY} / 47, \mathrm{XY},+18$ & IG \\
\hline & & $\{48, \mathrm{XY},+15,+18 / 47, \mathrm{XY},+15 /$ & $(25 \%: 75 \%)$ & \\
\hline & & $\begin{array}{l}47, \mathrm{XY},+18 / 46, \mathrm{XY}\} \\
(82 \%: 6 \%: 9 \%: 3 \%)\end{array}$ & & \\
\hline \multirow[t]{2}{*}{11} & $3714^{6 / 7}$ & Edad materna & $46, \mathrm{XX} / 47, \mathrm{XX},+\operatorname{mar}^{(\mathrm{a})}$ & CO: confirmado \\
\hline & & & $(45 \%: 55 \%)$ & IG \\
\hline \multirow[t]{2}{*}{12} & $4014^{1 / 7}$ & Edad materna $+\mathrm{VC}$ & $46, X Y / 47, X Y,+\operatorname{mar}^{(a)}$ & CO: confirmado \\
\hline & & $\begin{array}{l}\{46, X Y / 47, X Y+m a r\} \\
(75 \%: 25 \%)\end{array}$ & & IG \\
\hline \multirow[t]{2}{*}{13} & $3513^{0 / 7}$ & Edad materna & $46, X X / 46, X,+\operatorname{mar}^{(a)}$ & CO: $46, \mathrm{XX}$ \\
\hline & & & (92\%: 8\%) & RN normal \\
\hline \multirow[t]{2}{*}{14} & $3814^{0 / 7}$ & Edad materna & $\begin{array}{c}47, \mathrm{XX},+\operatorname{der}(22) \mathrm{t}(17 ; 22) \\
\text { mat }^{(\mathrm{b})}\end{array}$ & $\mathrm{CO}$ : confirmado \\
\hline & & & & IG \\
\hline 15 & $3114^{2 / 7}$ & $\begin{array}{l}\text { Hijo anterior con síndrome de } \\
\text { Down }\end{array}$ & $47, \mathrm{XY},+\mathrm{mar}^{(\mathrm{c})}$ & RN normal \\
\hline 16 & $4113^{1 / 7}$ & Edad materna & 47, XXY & IG \\
\hline 17 & $4114^{3 / 7}$ & Edad materna & 47, XXY & IG \\
\hline 18 & $3717^{0 / 7}$ & Edad materna & 47, XXY & IG \\
\hline \multirow[t]{2}{*}{19} & $4012^{5 / 7}$ & Edad materna + VC & 47, XXX,9qh+ & IG \\
\hline & & $(45, \mathrm{X}, 9 \mathrm{gh}+)$ & & \\
\hline \multicolumn{2}{|c|}{ TNA: Translucencia nucal aumentada } & IG: Interrupción del embarazo. & \multicolumn{2}{|c|}{ (b)Madre 46,XX,t $(17 ; 22)(\mathrm{p} 13 ; \mathrm{q} 11.2)$} \\
\hline \multicolumn{2}{|c|}{$\begin{array}{l}\text { HAM: Hijo anterior con } \\
\text { malformaciones }\end{array}$} & RN: Recién nacido & \multirow{2}{*}{\multicolumn{2}{|c|}{ (c) Padre 46,XY/47,XY,+mar }} \\
\hline CO: cordocen & & (a) Cariotipo normal de los padres & & \\
\hline
\end{tabular}


Tabla 5. Rearreglos cromosómicos equilibrados.

\begin{tabular}{|c|c|c|c|}
\hline Paciente & $\begin{array}{c}\text { Edad materna y } \\
\text { gestacional }\end{array}$ & INDICADOR & $\begin{array}{c}\text { REARREGLO CROMOSOMICO } \\
\text { EQUILIBRADO (AP) }\end{array}$ \\
\hline 20 & $4113^{2 / 7}$ & Edad matrena & $46, X Y, t(16 ; 17)(q 24 ; q 12)$ pat \\
\hline \multirow[t]{2}{*}{21} & $2313^{0 / 7}$ & Mosaico en VC & 45,XY,der(13;21)(q10;q10)mat \\
\hline & & $\begin{array}{l}\text { 45,XY,der(13;21)(q10;q10)/46,XY,(13;21) } \\
(\mathrm{q} 10 ; \mathrm{q} 10),+6\end{array}$ & \\
\hline \multirow[t]{2}{*}{22} & $3513^{2 / 7}$ & Edad materna + rearreglo & $46, X Y, \operatorname{inv}(10)(p 11 q 11) m a t$ \\
\hline & & $\begin{array}{l}\text { Cromosómico en la madre }\{46, X X, \operatorname{inv}(10) \\
\text { (p11q11)\} }\end{array}$ & \\
\hline
\end{tabular}

adicional era bisatelitado (presente en $54 \%$ de las metafases), se sugirió entonces el origen a partir de un cromosoma acrocéntrico. La cordocentesis realizada en la 20a semana confirmó el mosaicismo. Los padres presentaban cariotipo normal y optaron por la interrupción del embarazo.

Fue encontrado un marcador puntiforme en un $8 \%$ de las metafases provenientes de un tercer feto cuyo diagnóstico fue de mosaicismo 46,XX/46,X,+mar (paciente 13). Una cordocentesis fue realizada en la 20a semana para la confirmación de esos resultados; el resultado citogenético fue normal (46.XX). El examen ultrasonográfico, realizado en la 20a semana no reveló anormalidades. El estudio cromosómico de los padres fue normal. La gestación fue mantenida hasta el término, con el nacimiento de un niño normal.

Fue detectado cariotipo 47,XX,+mar en un cuarto caso (paciente 14). El cromosoma adicional satelitado era acrocéntrico, menor que los del grupo G. No fue identificado con las técnicas convencionales de coloración debido al tamaño reducido. Después del estudio cromosómico de los padres, se verificó un rearreglo heredado, hecho desconocido de la familia hasta entonces. Con las informaciones encontradas por el estudio del cariotipo materno, se verificó que el feto era [47,XX,+der(22)t(17;22)(p13;q11.2)mat]. La cordocentesis realizada en la 20a semana confirmó este resultado. El examen ultrasonográfico conducido en la 20a semana detectó arteria umbilical única. La pareja optó por la interrupción del embarazo.

El quinto caso envolviendo marcadores fue de un feto masculino (paciente 15). El cromosoma marcador era bisatelitado, metacéntrico, de menor tamaño que los del grupo G. Después de estudio cromosómico de los padres, se verificó presentar origen paterno (padre 46,XY/47,XY,+mar). Los resultados del examen ultrasonográfico fueron normales y la gestación fue mantenida hasta el término, con el nacimiento de un niño normal.

\section{Rearreglos equilibrados}

Tres rearreglos cromosómicos equilibrados fueron diagnosticados (Tabla 5): una inversión pericentrica (inv10), una translocación robertsoniana [der (13;21) (q10;q10)] — en este caso la amniocentesis se realizó para la confirmación de mosaicismo en $\mathrm{VC}-\mathrm{y}$ una translocación (16;17)(q24;q12). En los tres casos, los rearreglosfueron de origen parental. Las tres gestaciones evolucionaron normalmente.

\section{Confirmación de resultados de VC}

En algunos casos $(n=25)$, la AP fue realizada para la confirmación de mosaico en VC. Además de las líneas celulares normales, fueron encontradas líneas celulares anormales, así distribuidas: trisomías de los autosomos en 13 casos, aneuploidías de los cromosomas sexuales en cuatro, cromosomas marcadores en dos,tetraploidia en uno y rearreglos estructurales en cinco. De esos 25 casos, cuatro (16\%) fueron confirmados (Tabla 4): en

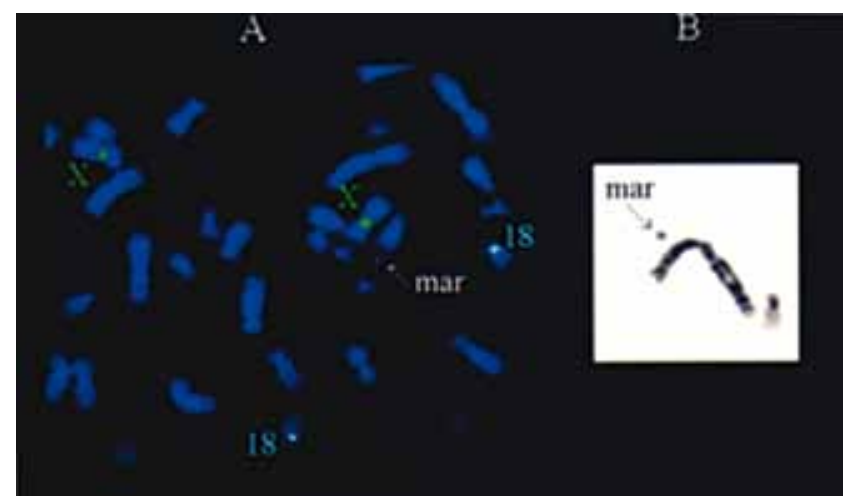

Figura 1: Resultados citogeneticos del feto con cariotipo 46,XX/,XX,+mar.

A: Cromosoma marcador (seta) después FISH (sonda AneuScreenVysis: DX1 SpectrumGreen, DYZ3 StectrumOrange, D18Z1 SpectrumAgua).

B: Cromosoma marcador (seta) después de bandas G. 

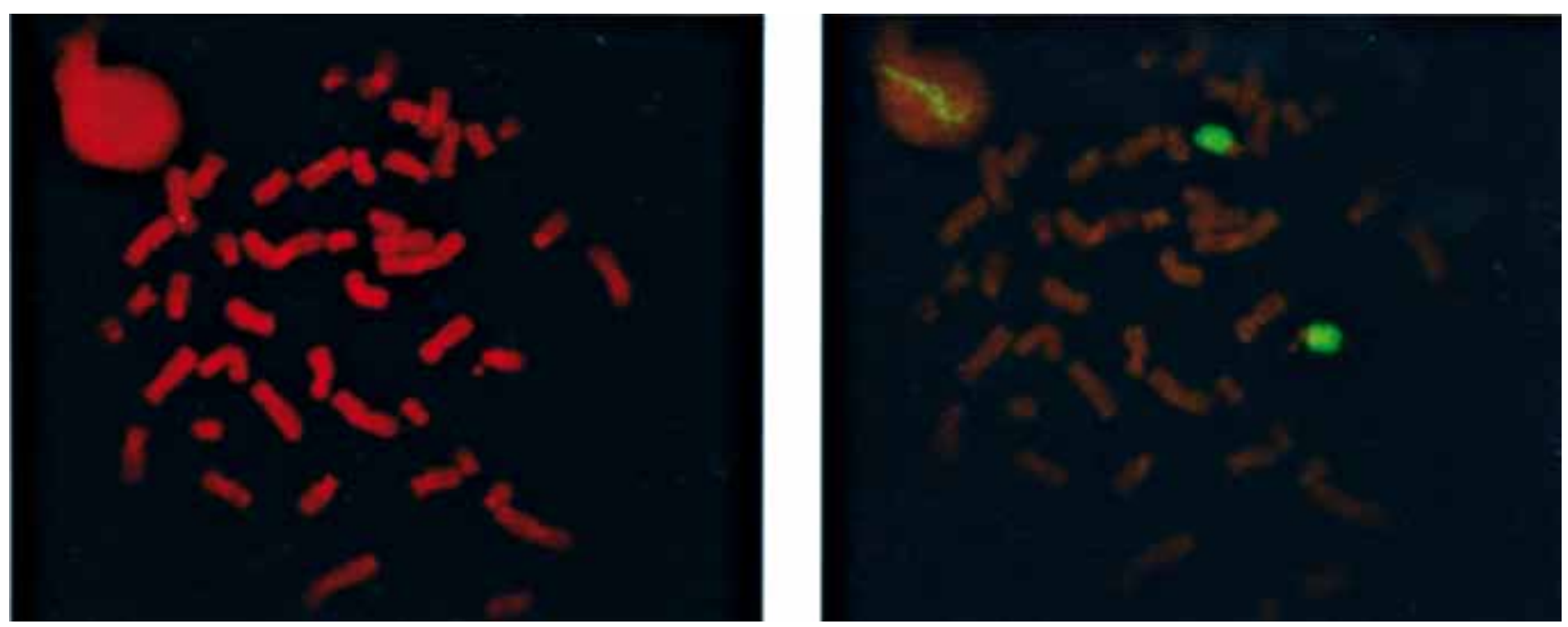

Figura 2: Metafase mostrando resultados de FISH del feto con cariotopo 46,XX/47,XX+mar. Sonda Coatsome (Oncor) para cromosoma 15.

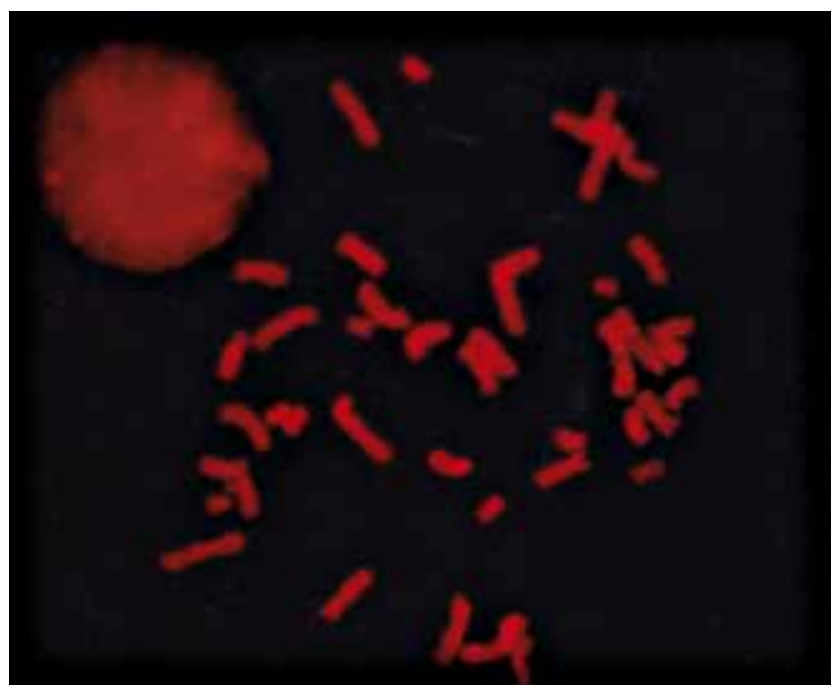

A
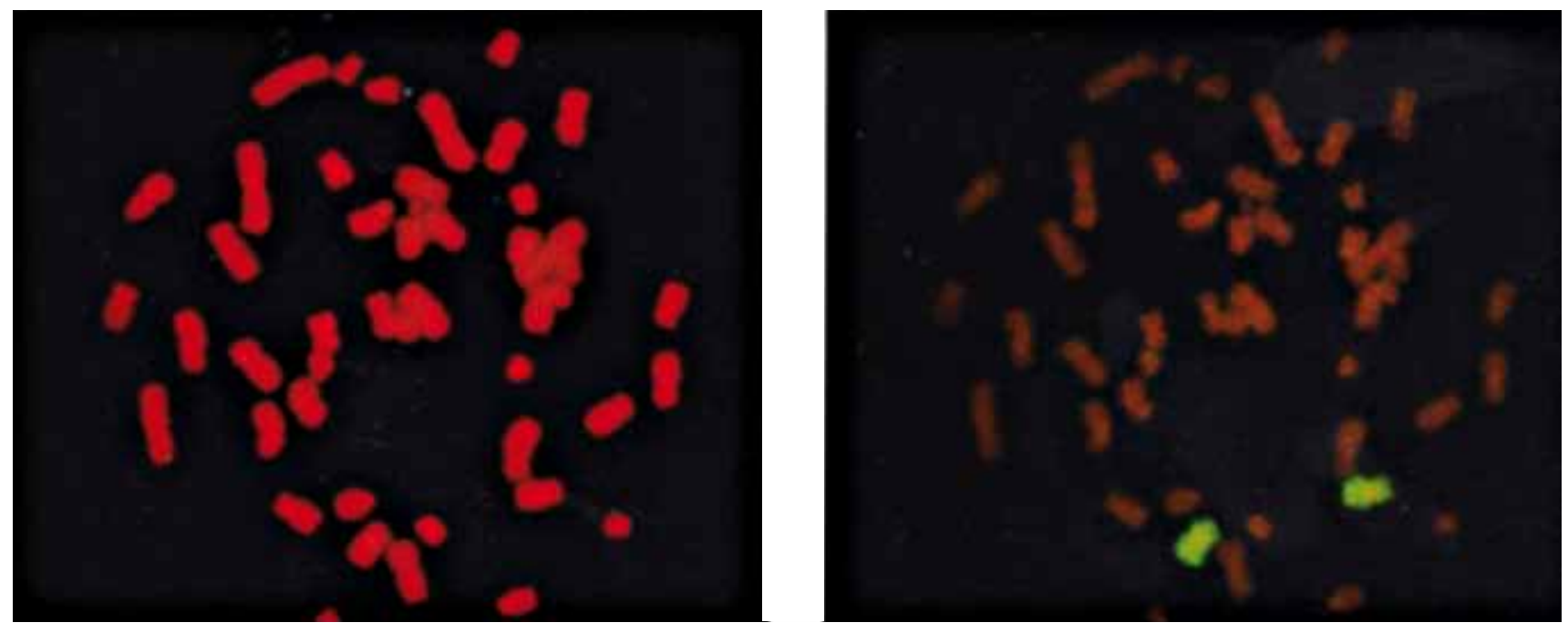

Figura 3: Metafase mostrando resultados de FISH del feto con cariotipo 46,XX/47,XX,+mar. Sonda Coatsome (Oncor) para los cromosomas 22 (A) y 9 (B). 
las gestaciones de las pacientes 10 y 12 fue confirmado el mosaicismo y en las pacientes 6 y 8 solamente la línea celular anormal fue confirmada.

En otros casos $(n=8)$, la AP fue realizada para la confirmación de resultados anormales en VC (no mosaico), o sea, dos casos de tetraploidía, uno de triploidía, dos de monosomía del cromosoma $\mathrm{X}$, dos de trisomía de los autosomos y un caso de rearreglo estructural. De estos ocho casos, dos (25\%) fueron confirmados (Tabla 4). En la paciente 1, fue confirmada la línea celular anormal y, en la 19, fue detectada unalínea celular anormal que no se había observado en el análisis de la VC.

\section{Pseudomosaicismo}

La frecuencia de pseudomosaicismo encontrada fue del 2,3\%, siendo $1,5 \%$ (ocho casos) de célula única y $0,8 \%$ (cuatro casos) de múltiples células. El hallazgo más frecuente fue la trisomía del cromosoma 2 (33\%). Se observaron tres casos $(0,6 \%)$ de contaminación por células maternas.

\section{Seguimiento}

La frecuencia de respuesta a los cuestionarios de seguimiento fue del 90,4\%. En 17 casos (3,2\%), hubo interrupción voluntaria de la gestación: una por anormalidad detectada a la ultrasonografía (anencefalia), una por razones sociales y 15 por aberraciones cromosómicas.

\section{Discusión}

El diagnóstico citogenético en muestras obtenidas por AP representa una alternativa en diversas situaciones en que es solicitado un estudio citogenético prenatal. La edad materna avanzada es la indicación más frecuente, llegando, en algunos servicios, a representar más del $70 \%$ de los casos $(10,11)$. En la población estudiada, $66,3 \%$ de las pacientes tenían esa indicación, presentando una edad de 38,3 $\pm 2,41$ años $(\mathrm{m} \pm 1$ d.p.). Ya está bien establecido que el riesgo de concebir un feto con aberraciones cromosómicas aumenta en el transcurrir de la vida reproductiva de la mujer a punto de que, después de 35 años, se justifica ofrecer a las pacientes exámenes citogenéticos prenatales. En este estudio, se encontraron diez cariotipos anormales (3,5\%) en 286 casos de exámenes citogenéticos realizados porque la embarazada presentaba 35 ańos o más. Sin embargo, en 24.901 amniocentesis de segundo trimestre estudiadas por Caron (11), realizadas por la indicación de edad materna > 35 ańos, la frecuencia de aberraciones cromosómicas fue del 1,8\%. El hecho puede ser atribuido a las diferentes épocas de realización de los procedimientos en cada estudio.

Además de la edad igual o superior a 35 años, otros factores de riesgo pueden aumentar la probabilidad de aberraciones cromosómicas en fetos. En el grupo estudiado, varias embarazadas se encuadraban en esa situación: en dos diagnósticos de trisomía del cromosoma 21, la ultrasonografía de primer trimestre había detectado translucéncianucal aumentada. La literatura confirma la relación entre marcadores ultrasonográficos, como la translucencianucal, y las anomalías cromosómicas $(12,13,14)$. En una paciente con historia de hijo anterior con malformaciones múltiples (sin que se haya realizado estudio cromosómico) el examen detectó feto $47, \mathrm{XX},+18$. Un feto con inversión $46, \mathrm{XY}, \operatorname{inv}(10)$ (p11q11)mat fue detectado en una madre con el mismo rearreglo cromosómico.

La frecuencia total de anomalías cromosómicas encontradas $(4,2 \%)$ es compatible con otros hallazgos de la literatura sobre AP, aunque algunos estudios relatan frecuencias más bajas: un $0,7 \%$ (15), un $1,9 \%$ (16), un $2,6 \%(17,18,19)$, un $3,3 \%$. (20). Sin embargo, la frecuencia referida en otros trabajos fue del $4,9 \%(2,21)$, un $5,5 \%$ (22) y un 6,2\% (10). La discrepancia entre los diferentes estudios es debida a la heterogeneidad de las poblaciones estudiadas, inclusive en lo concerniente a las indicaciones para el estudio cromosómico prenatal.

El diagnóstico de rearreglos cromosómicos aparentemente equilibrados y de pequeños cromosomas marcadores constituye una situación de difícil conducta en consejería genética, pues requiere cuidadosa evaluación citogenética e interpretación del significado clínico. Es de gran importancia obtener el cariotipo de los padres para determinar si el rearreglo fue heredado o expresado de nuevo. En los rearreglos equilibrados, si uno de los padres es portador del mismo, no hay evidencia de riesgo aumentado para 
anomalías fenotípicas o retardo mental. Sin embargo, se debe considerar la posibilidad que estos incluyan una anormalidad submicroscópica, no diagnosticable por la citogenética tradicional (23). Tres $(0,6 \%)$ rearreglos heredados (dos translocaciones equilibradas y una inversión) fueron diagnosticados en este estudio. Frecuencias semejantes fueron observadas por otros autores $(24,25)$. En los tres casos, el embarazo evolucionó normalmente hasta el término y los niños han presentado desarrollo cognitivo y psicomotriz normal para la edad.

Establecer un pronóstico enfrenta serias dificultades en el caso de cromosomas marcadores, cuya prevalencia en líquido amniótico varía de $0,05 \%$ a $0,11 \%(26,11$, 27). Cinco casos (un 0,9\%) fueron observados en la muestra evaluada, tres de ellos en forma de mosaico. Los estudios por bandeo G, Cy NOR no permitieron la identificación del origen de ninguno de los cromosomas marcadores encontrados. En uno de los casos, fue realizado un análisis por FISH que tampoco fue conclusivo en cuanto al origen del marcador.

Los hallazgos de cromosoma marcador en forma de mosaico demandaron el análisis de los tres frascos de cultivo para establecer el porcentaje real de mosaicismo. Laconsejería genética, se dio con base en datos existentes en la literatura. Se aconsejó también a las pacientes que confirmaran el diagnóstico citogenético a través del análisis de sangre fetal (cordocentesis) y seguimiento ultrasonográfico. Es importante resaltar que en los tres casos confirmados por cordocentesis los padres decidieron por la interrupción de la gestación. En el caso no confirmado, la pareja optó por continuar con la gestación, que prosiguió hasta el término, con un recién nacido normal. La confirmación del diagnóstico es esencial pues suministra datos que podrá ayudar en la decisión de la pareja con relación al mantenimiento o no del embarazo.

No es bien conocido el riesgo de alteraciones fenotípicas cuando un cromosoma marcador es identificado en el feto. En algunas familias, los cromosomas marcadores segregan sin causar cualquier efecto clínico aparente. Por otro lado, su presencia puede asociarse a malformaciones, compromiso mental, alteraciones del desarrollo sexual o problemas de fertilidad (28). Cuando el cromosoma marcador encontrado en el feto es heredado, normalmente no hay riesgo aumentado para anomalía fenotípica, salvo en los casos de progenitor en mosaico. Sin embargo, cuando el marcador es un pequeńo cromosoma heredado de un progenitor con translocación equilibrada, casi siempre se observan anomalías fenotípicas (29,23). Para los casos de novo, los riesgos fetales no son fáciles de cuantificar. Según Warburton (30), el riesgo de anomalía fenotípica es del 14,7\% en feto portador de cromosoma marcador no satelitado y de 10,9\% cuando el marcador es satelitado. El riesgo total para ambos tipos de marcadores es del $13,5 \%$. No se ha observado diferencias en el riesgo para anomalías fenotípicas cuando los fetos son portadores de cromosomas marcadores en mosaico.

Otro problema en el diagnóstico citogenético prenatal es el hallazgo de mosaicismo. Datos recolectados a partir de 179.663 amniocentesis mostraron una frecuencia del 0,309\% (555/179.663) de mosaicismo verdadero (31). Esos autores observaron que la forma más frecuente envolvió alteraciones numéricas de los cromosomas sexuales; mosaicismos envolviendo aberraciones estructurales de estos cromosomas (excluyéndose marcadores) fueron los menos frecuentes. En nuestra muestra encontramos cuatro mosaicos $(0,8 \%)$; tres de ellos incluían cromosoma marcador (anteriormente descritos). Como existen pocos datos sobre el seguimiento a largo plazo de nacidos vivos con mosaicismo, la consejería genética prenatal merece cautela. A los padres, se les debe explicar que la proporción de cada línea celular en cultivo no refleja la verdadera composición del mosaicismo en los tejidos fetales. A los padres se les debe dejar claro en cuanto a la imposibilidad de predecirse el fenotipo del feto. A lo sumo, es posible suministrar un espectro de los fenotipos posibles.

Tradicionalmente, se utilizada sangre fetal para confirmar o excluir el mosaicismo detectado en amniocitos. Conjuntamente con el examen ultrasonográfico, esa medida puede ser de gran auxilio en la decisión a ser tomada por los padres. Sin embargo, el ultrasonido presenta limitaciones y la sangre es solo uno de los varios tejidos fetales. Por eso, se debe tomar el cuidado de no proporcionar a los padres una falsa sensación de seguridad cuando el examen ultrasonográfico es negativo y no se encuentran células anormales en sangre fetal. La 
expresión variable de la anomalía en diferentes tejidos puede llevar a un diagnóstico prenatal incorrecto. El cariotipo normal en sangre fetal no excluye la posibilidad de mosaicismo verdadero en el feto (31, $32,33)$. Esos autores proponen que la biopsia de piel fetal debe ser utilizada en algunos tipos de mosaicismo en líquido amniótico.

El pseudomosaicismo, fenómeno de ocurrencia in vitro, no posee significado clínico, ni refleja el cariótipo fetal. Puede presentarse de dos formas: pseudomosaicismo de célula única, cuando la anormalidad aparece en una célula (mosaicismo nivel I) y el pseudomosaicismo de múltiples células, que se presenta en dos o más células en el mismo frasco de cultivo o en una única colonia en el caso del método de cultivo in situ (mosaicismo nivel II). En los dos casos, los resultados no se repiten en los otros frascos de cultivo $(34,8,35,36)$. La frecuencia de pseudomosaicismo en nuestra casuística fue de 2,3\%. Frecuencias menores han sido relatadas: $0,65 \%$ (37), un $1,75 \%$ (38). Sin embargo, en el trabajo del CEMAT, Winsor (16) reportaron 162 casos de mosaicismo nivel I $(7,8 \%)$ y 34 casos de mosaicismo nivel II (1,6\%). Kerber y Held, (39) relataron una frecuencia de pseudomosaicismo mayor en AP que en la AT. No se encontró explicación concreta para esos hallazgos, pues las condiciones de cultivo fueron idénticas en todas las muestras. Se presume que la presencia de un mayor porcentaje de células extraembrionarias en las muestras obtenidas en menor edad gestacional, podría ser la causa.

La frecuencia de contaminación por células maternas en las muestras analizadas fue del 0,6\%. Datos de cuatro grandes estudios colaborativos sugieren una frecuencia total del $0,25 \%$ de CCM en líquido amniótico $(40,41,42,43)$. Sin embargo, en algunos relatos la incidencia de contaminación llega a 0,5\% (8). Como generalmente es baja la proporción de células maternas y el análisis es siempre hecho en dos o más frascos de cultivo, raramente ocurren errores diagnósticos por ese motivo. Sin embargo, es importante implementar en cada laboratorio de diagnóstico prenatal, protocolos para la identificación de la CCM, teniendo en cuenta las graves consecuencias de resultados inexactos en el entorno prenatal,(44).

A pesar que el análisis cromosómico fetal por $\mathrm{VC}$ sea el método más rápido y precoz de diagnóstico fetal, en algunos casos es necesario efectuar una comprobación de los resultados a través de la amniocentesis, pues la presencia de líneas celulares anormales puede estar confinada a la placenta. En esos casos, la AP se torna una herramienta imprescindible para la confirmación precoz de los resultados de la VC. En nuestra muestra, fueron confirmados 18,2\% de los casos (6/33) de las anomalías cromosómicas en VC (en mosaico o no). En los casos adicionales, el cariotipo fue normal, confirmando que la anomalía estaba restricta a la placenta.

Varios puntos importantes del trabajo de laboratorio deben ser considerados cuando se utiliza AP para el diagnóstico de anomalías cromosómicas. La precisión del estudio citogenético en líquido amniótico es uno de los factores más relevantes. La calidad de los patrones de bandeo cromosómico de células obtenidas en cultivo de líquido amniótico de gestaciones precoces es semejante a aquella encontrada en AT (6).

El tiempo medio de colecta y entrega de los resultados varió en función del volumen de líquido amniótico aspirado, como se confirmó por el análisis estadístico de regresión lineal. El volumen de líquido amniótico utilizado para iniciar la cultura depende del protocolo utilizado. En nuestra experiencia, aproximadamente $1 \mathrm{ml}$ de líquido amniótico por semana gestacional fue recolectado. El volumen de muestra recibido fue dividido en dos alícuotas de igual volumen para iniciar tres cultivos primarios que normalmente avanzan a un ritmo ligeramente diferente; así, es posible procesarlos en días distintos. La elección del medio apropiado y la optimización de las condiciones de cultivo son importantes requisitos para minimizar el tiempo y la entrega de los resultados citogenéticos.

Cuanto mayor la edad gestacional en el momento de la colecta, mayor la probabilidad de éxito del cultivo y obtención del cariotipo. Existen más dificultades en el cultivo celular en muestras obtenidas antes de la 11a semana debido al menor número de células obtenidas. En esa edad gestacional, el crecimiento celular es lento. La mayoría de los estudios han publicado una tasa de éxito de 98 a 100\% en el cultivo de muestras recolectadas a partir de la 13a semana de gestación $(2,3)$. La tasa del $98,7 \%$ de éxito encontrada en nuestro estudio se asemeja a la descrita en la literatura. 
Con procedimientos de rutina bien establecidos y protocolos adecuadamente estandarizados en el laboratorio de diagnóstico prenatal, el cultivo de células de líquido amniótíco obtenido por AP no representa problemas. Con el perfeccionamiento en las técnicas de laboratorio, el éxito aumentó significativamente y se ha reducido substancialmente el tiempo medio de entrega de resultados.

Es importante destacar que varios centros de diagnóstico prenatal están disminuyendo el volumen de líquido amniótico recolectado por AP para minimizar los riesgos de problemas relacionados al procedimiento $(17,45,46)$. En nuestro servicio, la $\mathrm{AP}$ ya no es realizada en gestaciones con menos de 13 semanas y se hace un esfuerzo en conseguir resultados a partir de cantidades cada vez menores de líquido amniótico. En el momento, la cantidad necesaria de líquido amniótico para la obtención de resultados citogenéticos es de aproximadamente $10 \mathrm{ml}$ a las 13 semanas. Los resultados acerca de la tasa de éxito con estas condiciones, así como el tiempo de entrega del diagnóstico, están siendo analizados y serán publicados en un próximo informe.

\section{Agradecimientos}

Este trabajo hace parte de la tesis de doctorado Universidad de Sao Paulo. Beca del "Conselho Nacional de Desenvolvimento Científico e Tecnológico (CNPq)" Brasil.

\section{Refrerencias}

1. Jorgensen F.S., Bang J., Lind A.M., Christensen B. (1992).genetic amniocentesis at 7-14 weeks of gestation. Prenat.Diagn. 12:277-283.

2. Lockwood D.H., Neu R.L (1993). Cytogenetic analyses of 1375 amniotic fluid specimens form pregnancies with gestational age less than 14 weeks. Prenat.Diagn., 13:801-805.

3. Schulman L.P., Elias 8., Phillips O.P., Grevengood C., Dungan J.S., Simpson J.L. (1994). Amniocentesis performed at 14 weeks' gestation or earlier. Comparison with first-trimester transabdominal chorionic villus sampling.Obstet. GynecoL, 83:543-548.

4. Daniel A., NG A., kuah K.B., Reiha S., Malafiej P. (1998). A study of early amniocentesis for prenatal cytogenetic diagnosis.Prenat .Diagn., 18(1):21-28

5. Gollop T.R., Naccache N.F., Pieri P.C., Frigerio M.V., Zanforlin S.M (1994). Amniocentese Precoce com resultados rápidos: Análise de 167 casos. Livro de abstracts, II Encontro Nacional de Medicina Fetal. Belo Horizonte.

6. Nacache N., Jehee F., Bernal L.M., Gollop T.R (1996). Amniocentese Precoce entre 12 e 15 semanas de gestaçâo. Relato de 328 casos. Revista da Sociedade Brasileira de Medicina Fetal., 2:35-39.
7. Henry G.P., Miller W.A., 1992. Early Amniocentesis. J. Reprod. Med. 37(5):396-402.

8. Hus L.Y.F., Kaffe S., Jenkins E., Alonso L., Benn P.A., David K., Hirschhom K.,Lieber E., Shanske A., Shapiro L.R., Schutta E., Warbunton D. (1992). Proposed guidelines for diagnosis of chromosome mosaicism in amniocytes based and data derived for chromosome mosaicism and pseudomosaicism studies. Prenat.Diagn. 12(7):555-573.

9. Hus L.Y.F., Benn P.A. (1999). Revised guidelines for the diagnosis of mosaicism in amniocytes.Prenat. Diagn. 19:1081-1082.

10. Fernández J.M., Diaz J., Sanz R., Ramos C. (1997). Biopsia corial y amniocentesis en el primer trimestre. Situación actual. Prog. Diagn. Prenat., 9(9):500-509.

11. Caron L., Tihy F., Dallaire L. (1999). Frequencies of chromosomal abnormalities at amniocentesis: Over 20 year of cytogenetic analyses in one laboratory. Am. J. Med. Genet., 82:149-154.

12. Karaoguz MY, Bal F, Yakut T, et al . Cytogenetic results of amniocentesesismaterials : incidence of abnormal karyotypes en the Turkish collaborative study. Genet Counsel . 2006;17:219-30.

13. NeagosDaniela, CretuRuxandra; Sfetea Roxana Corina, BohilteaLaurentiuCamil.The importance of Screening and Prenatal Diagnosis in the Identification of the Numerical ChomosomalAbnormalities. A Journal of Clinical Medicine, Volume $6 \mathrm{~N}^{\circ} .32011$.

14. Gaudry Patrice, MaximeEsvan, Jean-Baptiste Meynard, Aziza Lebbar, AnésChoiset, Fanny Lewin, VassiliTsatsaris, Emmanuelle Pannier, Gilles Grange. Amniocentesis Performed for Karyotyping after Identified Ultrasonographic Abnormalities: What to Expect?. Fetal Diagn Ther 2012; 3:55-62.

15. Sundberg K, Lundsteen C., Philip J. (1999). Comparison of cell cultures, chromosome quality and karyotypes obtained after chorionic villus sampling and early amniocentesis with filter technique. Prenat.Diagn., 1912-16.

16. Nicolaides K.H., Brizol M.L., Patel F., Snijders R.J (1996). Comparison of Chorion Villus Sampling And early Amniocentesis for karyotyping in 1492 singlentonpregnanacies.Fetat.Diagn.Ther., 11:9-15.

17. Eiben B., Hammans W., Hansen S., Trawicki W., Osthelder B., Stelzer A., Jaspers K.D., Goebel R. (1997). On the complication risk of Early Amniocentesis versus Standard Amniocentesis.Fetal.Diagn.Ther., 12:140-144.

18. The Canadian Early and Mid-Trimester Amniocentesis Trial (CEMAT) Group. (1998). Randomised trial to asses safety and fetal outcome of early and midtrimester amniocentesis. Lancet, 351:242-247.

19. Winsor E.J.T., Tomkins D.J., Kalousek D., Farrel S., Wyatt P., Fan Y., Carter R., Wang H.,Dallaire L., Eydoux P., Welch J.P., Dawson A., Lin J.C.C., Singer J., Johnson JA, Wilson D. (1999). Cytogenetic aspects of the Canadian Early and Mid-trimester Amniotic Fluid Trial (CEMAT). Prenat.Diagn., 19:620-627.

20. Tharmaratnam S., Sadek S., Steele E.K., Harper M.A., Stewart F.J., Nevin J., NevinN.C.,Doman J.C. (1998). Early amniocentesis: Efect of removing a reduced volume of amniotic fluid on pregnancy outcome. Prenat.Diagn., 18:773-778.

21. Johnson J.M., Wilson R.D., Winsor E.J., Singer J., Dansereau J., Kalousek D.K (1996). The Early Amniocentesis Study: A randomized clinical trial of early amniocentesis versus midtrimesteramniocentesis.Fetat. Diagn. Ther. 11:85-93.

22. Farrán I., Pla F., Mediano C., Canet Y., Carreras E., de la Riva A.M. Cabero L. (1993). Amniocentesis precoz: Resultados en 206 casos con seguimiento neonatal. Prog. Diagn.Prenat., 5:73-78.

23. Gabarrón J., López I. (1998). Citogenética clásica : avances y limitaciones. Prog. Diagn.Prenat., 10(3):83-88.

24. Joffe R. (1989). Diagnóstico citogenético pré-natal através da amniocentese em gestantes com 35 anos ou mais de idade. Tese de Doutoramento, departamento de Biologia, Instituto de Biociências da Universidade de São Paulo. São Paulo.

25. Plasencia A., Hemando I., Benavides A., Coto E., FemándezToral J. (1996). Frecuencia de cromossomopatías balanceadas en la población detectada a partir de estudios prenatales. Prog.Diagn.Prenat, 8(3):127-130. 
26. Blennow E., Bui T.H., Kristoffersson U.,Vujie M. Annerén G., Holmberg E., Nordenskjold M. (1994). Swedish survey on extra structurally abnormal chromosomes in 39.105 consecutive prenatal diagnosis: prevalence an characterization by fluorescence in situ hybridization. Prenat.Diagn., 14:1019-1024.

27. Li M.M., Howard-Peebles P.N.., Killos L.D., Fallon L., listgarten E., Stanley W.S. (2000). Characterization and clinical implications of marker chromosomes identified at prenatal diagnosis.Prenat. Diagn. 20:138-143.

28. Buckton K., Spowart G., Newton M.S., Evans H.J. (1985).Forty four probands with in additional "marker chromosome". Hum. Genet., 69:353-370

29. Stamberg J., Thomas G.H. (1986). Unusual supemumerary chromosome: types encountered in a referred population, and high incidence of associated matemal chromosome abnormalitíes. Hum. Genet, 72:140-14-4.

30. Warburton D. (1991). De Novo balanced chromosome rearrangements and extra marker chromosomes identified at prenatal diagnosis: Clinical significance and distribution of breakpoints, Am. J. Hum. Genet., 49:995-1013

31. Hus L.Y.F., YU M.T., Richkind K.E., Van Dyke D.L., Crandall B.F., Saxe D.F.,Khodr G.S., Mennuti M., Stetten G., Miller W.A., Priest J.H. (1996). Incidence and significance of chromosome mosaicism involving an structural abnormality diagnosed prenatally through amniocentesis: A collaborative study. Prenat.Diagn. 16:1-28.

32. Berghella V., Wapner R.J., Yang-FengT.,Mahoney M.J. (1998. Prenatal confirmation of true fetal trisomy 22 mosaicism by fetal skin biopsy following normal fetal blood sampling.Prenat. Diagn., 18:384-389.

33. Marques I. (1998). Mosaicismo em diagnostico pré-natal. Prog. Diagn. Prenat.,103: 89-92.

34. Krawczun M.S., Jenkins E.C.,Masia A., Kunapom S., Stark S.L., Dunca Ch., Sklower S.L., Rudelli R.D. (1989). Chromosomal abnormalities in amniotic fluid cell cultures: A comparison of apparent pseudomosaicism in Chang and RPMI-1649 media. Clin.Genet., 35:139-145.

35. Tejada M.I. (1994). Citogenética del líquidoamniótico.Posibilidadestécnicas, eficiencia y aspectoscontrovertidos.Prog.Diagn.Prenat, 6(2):105-118.
36. Verma R., Kleyman S., Conte R. (1998). Chromosomal mosaicisms during prenatal diagnosis. Gynecol. Obstet. Invest., 45:12-15.

37. Elejalde B.R., de Elejalde M.M., Acuna J.A., Thelen D., Trujillo C., Karrman M. (1990). Prospective study amniocentesis performed between weeks 9 and 16 of gestation. Its feasibility, risks, complications and use in early genetic prenatal diagnosis. Am. J. Med. Genet., 35:188-196.

38. Rebello M.T., Gray C.T., Rooney D.E., Smith J.H., Hacketl G.A., Loeffler F.E., HorvvellD.H.,Beard RYW., Coleman D.V. (1991). Cytogenetic studies of amniotic fluid taken before the 15th week of pregnancy for earlier prenatal.Prenat.Diagn., 11:35-40.

39. Kerber S., Helde K.R. (1993).Early genetic Amniocentesis-4 years' experience.Prenat.Diagn., 13(1): 21-27.

40. Benn P.A., Hus L.Y.F.(1983). Maternal cell contamination of amniotic fluid cell cultures: results of a US nationwide survey. Am. J. Med. Genet., 15:297-305.

41. Benn P.A., Schonhaut A.G., Hus L.Y.F.(1983). A high incidence of maternal cell contamination of amniotic fluid cell cultures: Am. J. Med. Genet., 14:361-365.

42. Bui T.H., Iselius L., Lindsten J. (1984). European collaborative study on prenatal diagnosis: mosaicismpseudomosaicism and single abnormal cell in amniotic fluid cell cultures. Prenat .Diagn.,(Special Issue) 4:145-162.

43. Worton R.G., Stem P. (1984). A Canadiam collaborative study of mosaicism in amniotic fluid cell cultures.Prenat.Diagn., (Spec.lss)., 4:131-144.

44. Laboratory Guidelines for Detection, Interpretation, and Reporting of Maternal Cell Contamination in Prenatal Analyses. The Journal of Molecular Diagnostics, Vol. 13, No. 1 January 2011.

45. Eiben B., Hammans W., Trawicki W., Goebel R. (1998).Early Amniocentesis versus Chorionic Villus Sampling. Prenat.Diagn. Letters to the editor., 18:405-406.

46. Schrijer I, Cherny SC, Zehnder JL: Testing for maternal cell contamination in prenatal samples: a comprehensive survey of current diagnostic practices in 35 molecular diagnostic laboratories. J MolDiagn 2007, 9:394-400
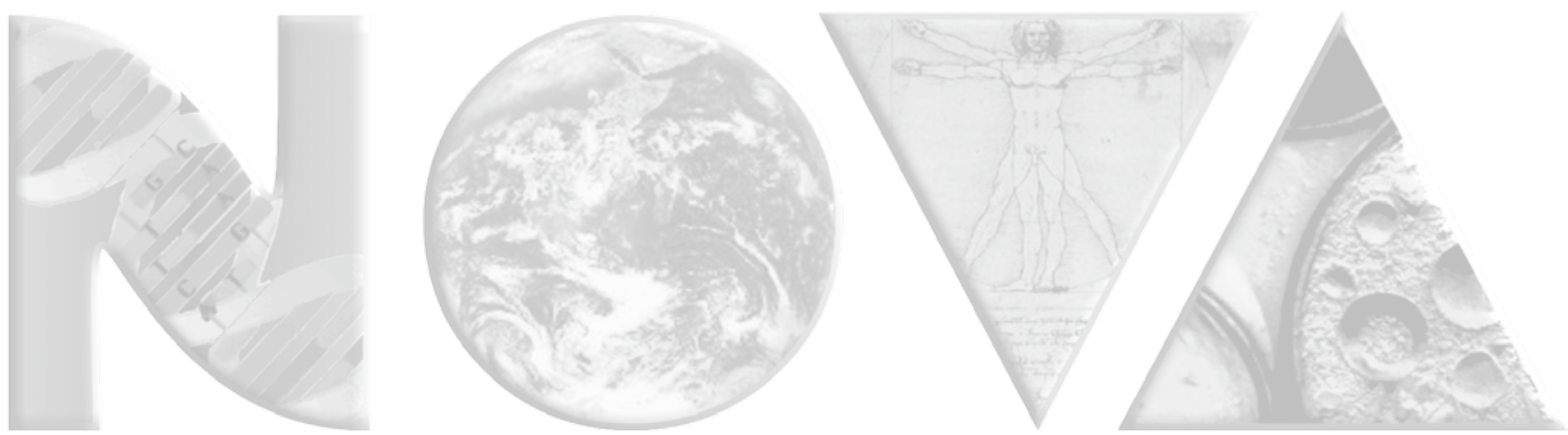